\title{
Lateral cutaneous nerve of the thigh injury during gynaecological laparoscopic procedure
}

\author{
G. Botros • J. J. O. Herod
}

Received: 28 November 2009 / Accepted: 6 January 2010/Published online: 9 March 2010

(C) Springer-Verlag 2010

\begin{abstract}
This is a case report of a woman who developed severe meralgia paraesthetica due to an injury to the lateral cutaneous nerve of the thigh. The injury occurred during a laparoscopy and adhesiolysis procedure as a result of the positioning of one of the secondary ports close to the inguinal ligament. Symptoms were reported immediately after the procedure with tingling and numbness of the upper lateral aspect of the right thigh. The symptoms persisted for more than 3 months and required strong regular analgesia. She continues to suffer significant neurological symptoms and is considering a nerve decompression procedure. The lateral cutaneous nerve of the thigh varies in its course in the abdominal wall. This is particularly variable while it crosses the inguinal ligament. Hence, any ports placed close to the inguinal ligament near to the anterior superior iliac spine may injure the nerve resulting in meralgia paraesthetica. Injury to the nerve has been reported in different types of procedures, e.g. laparoscopic inguinal hernia repair. We believe that it has not been reported previously after a gynaecological laparoscopic procedure. In this case report, we highlight the possibility of an injury to the lateral cutaneous nerve of the thigh during gynaecological laparoscopic procedures. We review the anatomy and the variations in the nerve's course that could lead to its injury during minimal invasive gynaecological operations.
\end{abstract}

Keywords Lateral cutaneous nerve of the thigh . Laparoscopy $\cdot$ Meralgia paraesthetica $\cdot$ Nerve injury

\footnotetext{
G. Botros $(\bowtie) \cdot$ J. J. O. Herod

Liverpool Women's Hospital,

Crown Street,

Liverpool L8 7SS, UK

e-mail: gbotros72@hotmail.com

J. J. O. Herod

e-mail: Jonathan.herod@1wh.nhs.uk
}

\section{Case report}

A 23-year-old woman was referred to the gynaecology clinic with a history of right iliac fossa pain. The pain was persistent and became worse during the time of menstruation.

She had a past history of an appendicectomy and a previous diagnostic laparoscopy 4 years earlier. She had never been pregnant and was using the combined oral contraceptive pill for contraception. There was no other significant past medical history.

After the initial gynaecological assessment, a provisional diagnosis was made of right iliac fossa pain due to either endometriosis or pelvic adhesions. The plan was made to proceed to diagnostic laparoscopy with adhesiolysis if required.

The patient agreed to have the laparoscopy procedure, and consent was obtained. A closed laparoscopy technique was performed. A pneumo-peritoneum was established via the per-umbilical insertion of the Veress needle. A $10-\mathrm{mm}$ primary trocar was inserted at the umbilicus. Two 5-mm ports were inserted in the iliac fossae. The two secondary ports were noticeably near the inguinal ligament close to the anterior superior iliac spines. A good view of the pelvis and abdomen was obtained. Filmy adhesions of the ascending and the descending colons were divided. The pelvic organs were found to be normal. No evidence of endometriosis or any other pathology was identified.

On the same night after the procedure, the patient complained of right-sided pains around the right secondary port site and numbness of the upper lateral thigh. She was prescribed oral analgesia and discharged home on the following day.

Eight weeks later, she was reviewed in the gynaecology outpatient clinic. She was complaining of persistent severe tingling and numbness of the antero-lateral aspect of the upper thigh. She had been prescribed pregabalin $75 \mathrm{mg}$ daily for this symptom by her general practitioner. No 
Fig. 1 Distribution of the lateral cutaneous nerve of the thigh (with permission from bartlebycom.com.Inc.)

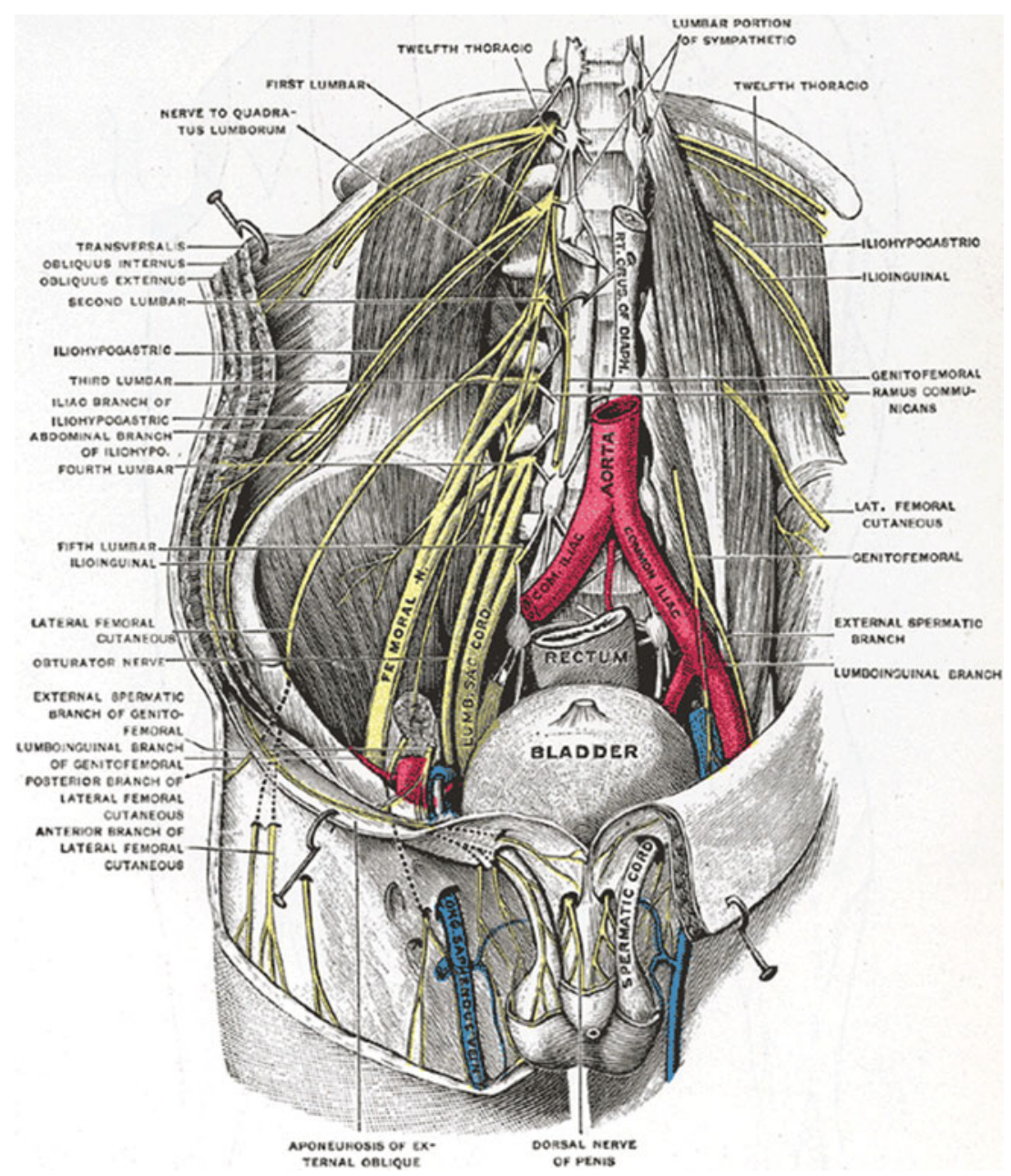

motor or other neurological symptoms or signs were elicited. She was referred to a neurologist for further evaluation and management.

Neurological examination demonstrated tenderness over the point of emergence of the lateral cutaneous nerve of the thigh and percussion of this area elicited paraesthesia over her numb thigh area (Tinnel's sign). She had altered pin prick, temperature, and light touch sensitivity in a distribution which correlates with the area supplied by the lateral cutaneous nerve (LCN) of the thigh. The diagnosis was meralgia paraesthetica due to LCN injury during the laparoscopic procedure upon insertion of the right lateral secondary port. Follow-up appointments over the following 6 months showed no improvement, and the patient is currently considering nerve decompression.

\section{Discussion}

The lateral cutaneous nerve of the thigh is a pure sensory nerve distributed only to the skin and arises from the lumbar plexus, from the second and third lumbar nerves. It

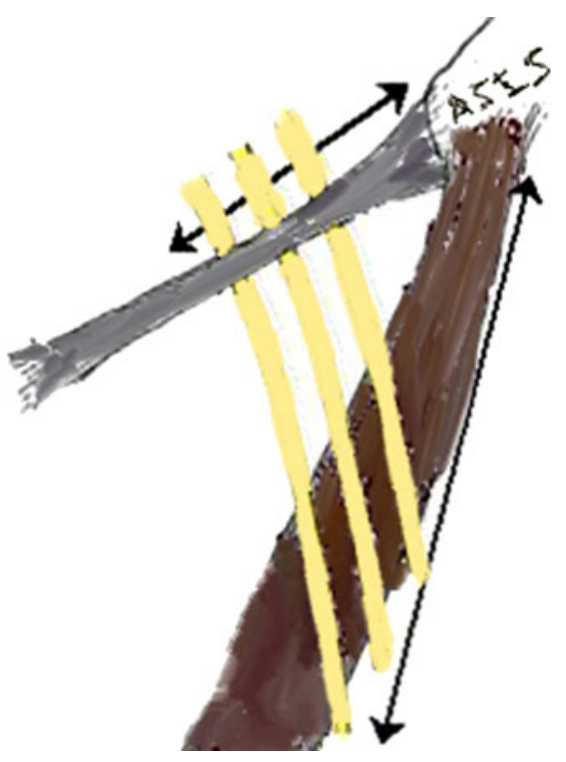

Fig. 2 Nerve crosses the inguinal ligament $6 \mathrm{~mm}$ to $7.3 \mathrm{~cm}$ medial to ASIS and lateral border of Sartorius 2.2 to $11.3 \mathrm{~cm}$ distal to the ASIS 
emerges from the lateral border of the psoas major muscle and crosses the iliacus muscle, beneath the iliac fascia, to reach the anterior superior iliac spine (Fig. 1). It enters the thigh beneath the lateral end of the inguinal ligament and passes either over, under, or through the origin of the sartorius muscle. It extends distally along the front of the thigh for a few inches, lying at first beneath the fascia lata, and afterwards in a tubular investment of the fascia. It gives off small branches in this part of its course, and finally, piercing the fascia about four inches distal to the anterior superior iliac spine, it separates into anterior and posterior terminal branches. The anterior branch is the larger and is distributed on the lateral aspect of the front of the thigh almost to the knee. The smaller posterior branch supplies the skin of the lateral side of the buttock, distal to the greater trochanter, and the skin of the proximal two thirds of the lateral aspect of the thigh [1].

Variation in the course of this nerve while crossing the inguinal ligament is documented in the literature. Generally, it enters the thigh medial to the ASIS under the inguinal ligament. In about $30 \%$ of cases, the nerve divides before crossing the inguinal ligament. The nerve then proceeds laterally and crosses the lateral border of Sartorius at a variable distance from the ASIS. Generally, it crosses the inguinal ligament $6 \mathrm{~mm}$ to $7.3 \mathrm{~cm}$ medial to ASIS and lateral border of Sartorius 2.2 to $11.3 \mathrm{~cm}$ distal to the ASIS [2, 3] (Fig. 2). The most frequent non-surgical cause of damage to this nerve is entrapment at the level of the inguinal ligament. This is most often seen in association with obesity but also in other conditions that increase intraabdominal volume such as pregnancy and ascites, when the nerve may be kinked or compressed by the bulging abdomen as it leaves the pelvis. However, entrapment may also occur in lean subjects and in children [4].

The injury of the lateral cutaneous nerve due to surgery is reported after laparoscopic inguinal hernia repairs, iliac crest carving for bone graft operations, and wide low transverse abdominal incision. It has never been reported after gynaecological laparoscopic procedure. The positioning of the lateral port in this case was (for cosmetic reasons) low, close to the inguinal ligament, and laterally placed near to the anterior superior iliac spine. Secondary port positioning differs according to the type of laparoscopic procedure. The RCOG recommends that secondary port entries should be under vision and lateral to the obliterated umbilical artery to avoid injury to the inferior epigastric vessel [5]. Ideally for operative gynaecological laparoscopy, a satisfactory instruments relationship in the abdominal cavity includes an angle of $60^{\circ}$ between the two instruments' tips, tangential approach to the site, and appropriate working distance. Consequently, for most gynaecological laparoscopic procedures, the secondary ports are positioned in the iliac fossae lateral to the lateral border of the rectus abdominis muscle at the junction of the upper two thirds and the lower third of an imaginary line connecting the anterior superior iliac spine to the umbilicus. Positioning of the lateral port closer to the inguinal ligament or the anterior superior iliac spine increases the risk of injury to the lateral cutaneous nerve of the thigh.

Meralgia paraesthetica (Greek: Meros Algos, meaning thigh pain) is the name of the clinical syndrome caused by damage to the lateral cutaneous nerve of the thigh. This causes various sensory symptoms over the antero-lateral aspect of the thigh [6]. The diagnosis is easy to make on clinical grounds. The patient complains of varying degrees of pain, burning, numbness, paraesthesiae, and dysaesthesiae over the anterolateral aspect of the thigh. Rarely, confirmation is needed by nerve conduction studies, which may show a reduction in sensory conduction velocity and/or reduction in amplitude or absence of the response [7]. The condition may resolve on its own [8]. Therefore, reassurance may be the only advice needed, especially if the condition is mild. For more severe cases, other treatments are suggested including local anaesthetic infiltration and nerve block, decompression (neurolysis), and rarely neurectomy [6].

In conclusion, positioning of the secondary port in the iliac fossae can cause injury to the lateral cutaneous of the thigh especially if the ports are situated near the inguinal ligament and the anterior superior iliac spine. This is followed by immediate unpleasant sensory symptoms in the skin area supplied by the nerve. These symptoms in most cases resolve spontaneously over a period of 2-3 months. Rarely symptoms may persist, requiring further interventions.

Conflict of interest There is no actual or potential conflict of interest in relation to this article.

\section{References}

1. Anatomy of the lumber plexus. In: Cunningham's regional anatomy, 12 edn, p 722-724

2. Grothaus MC, Holt M, Mekhail AO, Ebraheim NA, Yeasting RA (2005) Lateral femoral cutaneous nerve: an anatomic study. Clin Orthop Relat Res 437:164-168

3. Crenshaw AH (1992) Surgical approaches: hip. In: Crenshaw AH (ed) Campbell's operative orthopaedics, 8th edn. Mosby Year Book, St Louis, pp 58-71

4. Edelson R, Stevens P (1994) Meralgia paresthetica in children. J Bone Joint Surg Am 76(7):993-999, MEDLINE: 94299589

5. RCOG (2008) Green-Top Guideline 49: preventing entry-related gynaecological laparoscopic injuries. RCOG Green Top Guidelines

6. Khalil N, Nicotra A, Rakowicz W (2008) Treatment for meralgia paraesthetica. Cochrane Database Syst Rev 16(3):CD004159

7. Lagueny A, Deliac MM, Deliac P, Durandeau A (1991) Diagnostic and prognostic value of electrodiagnostic tests in meralgia paraesthetica. Muscle Nerve 14:51-56. doi:1, MEDLINE: 91125398

8. Ecker AD, Woltman HW (1938) Meralgia paraesthetica: a report of one hundred and fifty cases. J Am Med Assoc 110:1650-1652 